\title{
Topological and Energetic Conditions for Lithographic Production of Carbon Nanotubes from Graphene
}

\author{
D. Fülep, ${ }^{1}$ I. Zsoldos, ${ }^{1}$ and I. László ${ }^{2}$ \\ ${ }^{1}$ Faculty of Technology Sciences, Széchenyi István University, Györ 9026, Hungary \\ ${ }^{2}$ Department of Theoretical Physics, Institute of Physics, Budapest University of Technology and Economics, Budapest 1521, Hungary \\ Correspondence should be addressed to I. Zsoldos; zsoldos@sze.hu
}

Received 21 April 2015; Accepted 2 July 2015

Academic Editor: Stefano Bellucci

Copyright @ 2015 D. Fülep et al. This is an open access article distributed under the Creative Commons Attribution License, which permits unrestricted use, distribution, and reproduction in any medium, provided the original work is properly cited.

Density Functional Based Tight-Binding (DFTB) molecular dynamics (MD) simulations were performed for producing carbon nanotubes from graphene nanoribbons. The constant temperature simulations were controlled with the help of Nosé-Hoover thermostat. In our systematic study we obtained critical curvature energies and determined topological conditions for nanotube production from two parallel graphene nanoribbons. We obtained linear relationship between the curvature energy and the square of the curvature.

\section{Introduction}

Although the exceptional electric properties of carbon nanotubes have already been proven in several publications [1], until now only very few electric devices were presented or realized [2-4]. This fact can be explained by the lack of well-controlled reliable technology for nanotube or nanotube network construction. Nanotube construction from nanoribbons is a promising possibility. Nanoribbons can be produced with the help of nanolithography [5] and various chemical compounds [6-8]. Special ribbons can be obtained by cutting of nanotubes [9]. The electronic properties of graphene nanoribbons are under intensive theoretical [10$13]$ and experimental $[13,14]$ study. Various ribbon structures as the $\mathrm{L}[15]$, the $\mathrm{T}[16-18]$, and the $\mathrm{Z}[19,20]$ structures were suggested for various nanoelectric building blocks. Experimental and simulational methods are used for the study of nanoelectric networks [21] and functional units [22-24]. For the time being the accuracy of nanoribbon cutting from graphene is about few $\mathrm{nm}$, and only one order of magnitude is missing to the atomic accuracy. There are studies for the instabilities at nanoribbon edges and nanotubes are obtained in molecular dynamics simulations from two nanoribbons $[25,26]$. It was demonstrated in molecular dynamics simulations that graphene patterns with atomic accuracy can develop in a self-organizing way to the predetermined fullerenes or nanotubes [27-29].

The importance of nanotube production from two nanoribbons comes from the fact that in this way open ended carbon nanotubes are developed, but the one-pattern nanotubes are always closed at one end [27]. Nanotube growing from nanoribbons is not a trivial task although the idea has already been published $[25,26]$. In the present work we shall study the topological and energetical conditions for the growing of perfect nanotubes. We shall study the connection between the curvature energy and the nanotube curvature.

\section{The Method}

The interatomic interaction was calculated with the help of Density Functional Tight-Binding method [30]. The nanoribbons were cut out from a graphene sheet of interatomic distance $r=1.42 \AA$. After putting the two nanoribbons one over the other with parallel position, the nanotube formation was simulated in a molecular dynamics calculation with constant environmental temperature [31,32]. The time step was $\Delta t=0.7 \mathrm{fs}$ and the Verlet algorithm [33] gave the velocity. The initial atomic displacements during the time step 


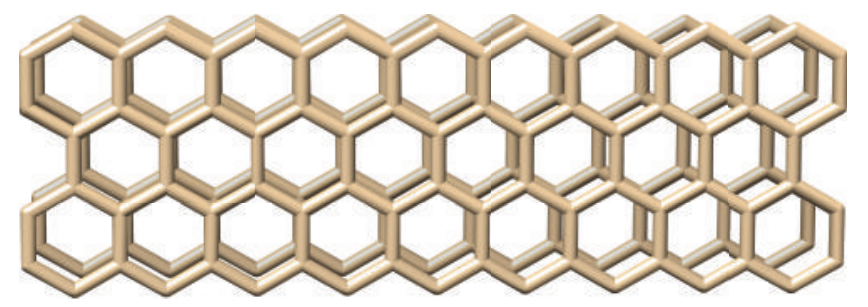

(a)

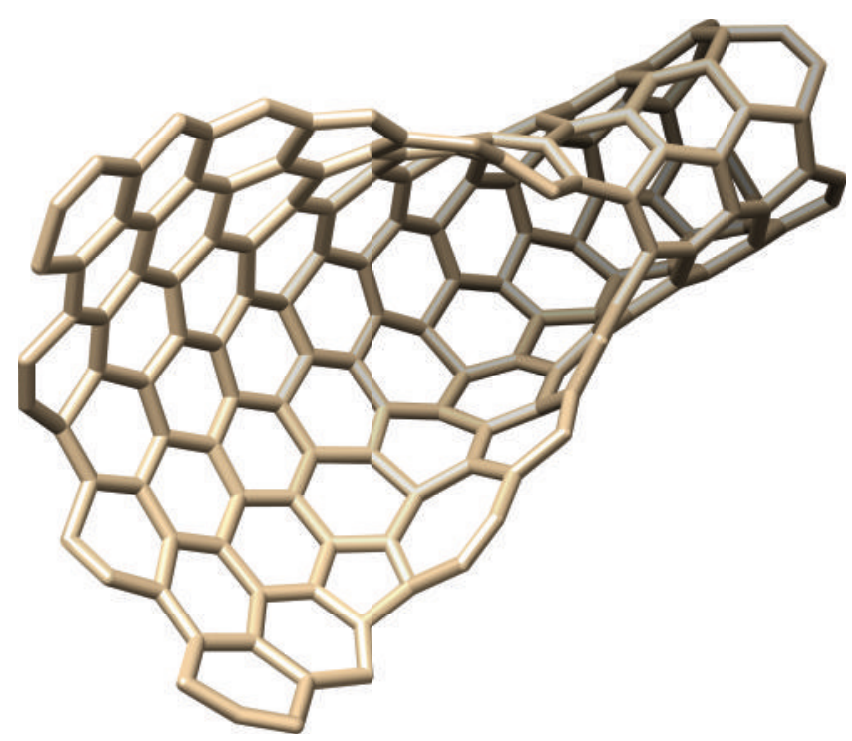

(b)

FIGURE 1: Simulation of armchair nanotubes. The initial (a) and the final (b) structures are shown. The simulation parameters are the following: $1000 \mathrm{~K}$ simulation temperature, $22.13 \AA$ of length, and $7.10 \AA$ of width for the parallel nanoribbons.

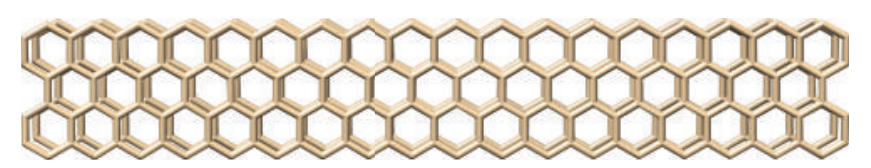

(a)

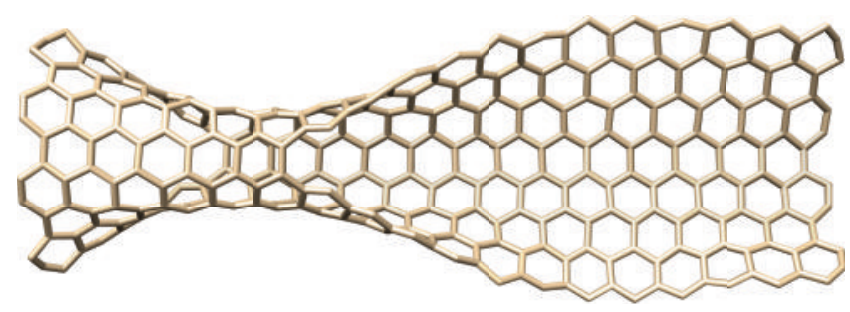

(b)

FIgURE 2: Simulation of armchair nanotubes. The initial (a) and the final (b) structures are shown. The simulation parameters are the following: $1250 \mathrm{~K}$ simulation temperature, $44.27 \AA$ of length, and $7.10 \AA$ of width for the parallel nanoribbons.

of $\Delta t=0.7 \mathrm{fs}$ were sorted randomly and they gave the initial velocities by appropriate scaling. In this scaling we supposed an initial kinetic temperature $T_{\text {init }}$. This initial temperature was chosen from the range of $T_{\text {init }}=1000 \mathrm{~K}$ and $1100 \mathrm{~K}$. We have found that the final structure was depending more strongly on the direction of the initial velocities than the actual value of $T_{\text {init }}$. That is, by scaling of the initial temperature in the above-mentioned range, the final structure was not strongly changing. As the formation of new bonds decreased the potential energy and increased the kinetic energy we had to keep the temperature constant. In a constant energy calculation the kinetic energy obtained by forming new bonds destroyed other bonds of the structure. We used Nosé-Hoover thermostat [31, 32, 34, 35] for the constant temperature simulation. It is evident that in the Nosé-Hoover thermostat there is an oscillation of the temperature but it can not destroy the structure formation. In the following the temperature of the calculation will mean the temperature of the thermostat. If the constant temperatures were realized with the help of random scaling of the kinetic energy we could not distinguish the temperature of the environment and the structure. This is why we can speak about the $T_{\text {init }}$ temperature and the temperature of the Nosé-Hoover thermostat (the environment temperature).

\section{Results}

We were studying armchair and zigzag nanotubes. The initial structure contained two congruence graphene nanoribbons put one over the other at a distance of $3.4 \AA$ (Figure 1). We calculated the interatomic forces between the carbon atoms and we were awaiting new bond formations at the edges of the ribbons. We wanted to obtain the predefined nanotube in a self-organizing process. According to our simulations the formation conditions were depending on the type of the nanotube. In the following we shall present our results for armchair and zigzag nanotubes.

3.1. Armchair Nanotubes. The basic problem of armchair nanotube formation can be seen in Figure 1. The simulation process of two congruence and parallel nanoribbons was 


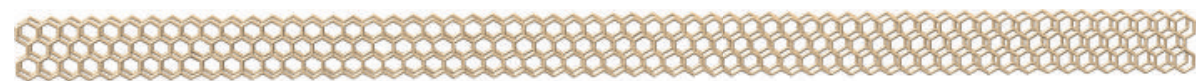

(a)

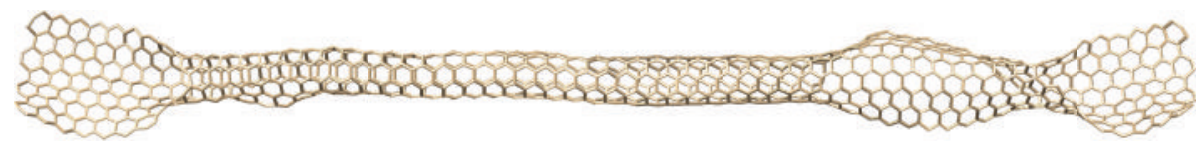

(b)

FIgURE 3: Simulation of armchair nanotubes. The initial (a) and the final (b) structures are shown. The simulation parameters are the following: $1100 \mathrm{~K}$ simulation temperature, $145.10 \AA$ of length, and $7.10 \AA$ of width for the parallel nanoribbons.

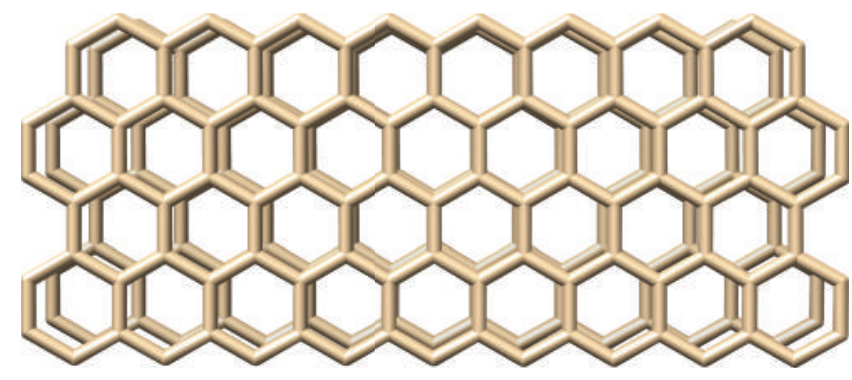

(a)

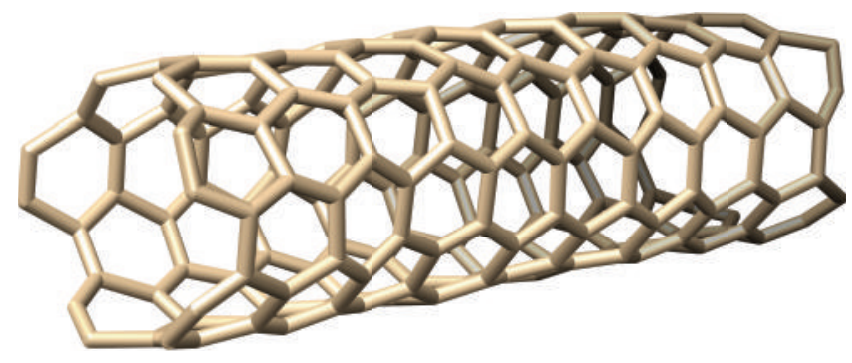

(b)

FIGURE 4: Simulation of armchair nanotubes. The initial (a) and the final (b) structures are shown. The simulation parameters are the following: $1000 \mathrm{~K}$ simulation temperature, $22.13 \AA$ of length, and $9.23 \AA$ of width for the parallel nanoribbons.

performed at $1000 \mathrm{~K}$ temperature. We observed the initial growing together at both sides of the ribbons, but the process stopped at the established structure of the figure. At one side there is a tendency to form a graphene sheet.

We repeated the experiment at lower and higher initial temperatures as well, but we did not obtain the desired structure. In the range of $(700 \mathrm{~K}, 1300 \mathrm{~K})$ the final structure was very similar to that of Figure 1 . At even lower temperatures the formation process could not start and at higher temperatures we obtained a complete destruction.

In order to yield a perfect nanotube we tried to change the size of the ribbons. First we increased the length as in this way we hoped to increase the probability to form favorable bond formation. We hoped as well that the increased number of new bonds could supply enough forces for the structure formation. Figures 2 and 3 show the new simulations. The increased lengths are taken at simulation temperatures of $1250 \mathrm{~K}$ and $1100 \mathrm{~K}$.

Although in Figures 2 and 3 the nanotube formation started at various parts of the ribbons the final structures are still frozen in structures with full of defects similar to Figure 1. Changing the temperatures we did not obtain perfect structures. Thus we decided to change the widths of the ribbons as well. In the following we shall present only the results of the most characteristic runs.

In Figure 4 we present the results of a simulation with increased width $(9.23 \AA)$ of the nanoribbons. The environmental temperature was $1000 \mathrm{~K}$. We can see that with increased ribbon widths we obtained perfect nanotube even at a short length.
Figure 5 shows that by increasing the width of the nanoribbon the formation of perfect nanotube will be kept.

Our results that the nanotube formation depends on the nanotube width can be explained with the help of the $E_{c}$ curvature energy:

$$
E_{c}=E_{\text {tube }}-E_{\text {graphene }}
$$

Here $E_{\text {tube }}$ and $E_{\text {graphene }}$ are the formation energies of nanotube and graphene. These energies are normalized to atoms. Figure 6 shows the $E_{c}$ curvature energies in the function of the nanotube radius. We can see that the curvature energy decreases as the radius increases. There is a critical curvature energy over which the heat energy of the environment can not produce the energy sufficient for overtaking energy barrier of the bond formation. By increasing the temperature the structure could overtake this barrier, but it could destroy the other bonds as well. Figure 6 shows that the formation of nanotube can happen only if the corresponding curvature energy is less than critical curvature energy of $0.18 \mathrm{eV}$. This critical curvature energy corresponds to the nanotube $(5,5)$ of radius $3.3 \AA$ which is obtained from the ribbons of widths $9.23 \AA$ (Figure 4).

3.2. Zigzag Nanotubes. In the case of zigzag nanotubes there is an extra complication. If we put two congruence and parallel zigzag nanoribbons one over the other, the final structure will be that of Figure 7. Instead of obtaining a zigzag nanotube we obtained an armchair one rotated by $90^{\circ}$ degrees. Namely, the atoms to be bonded at the zigzag side of 


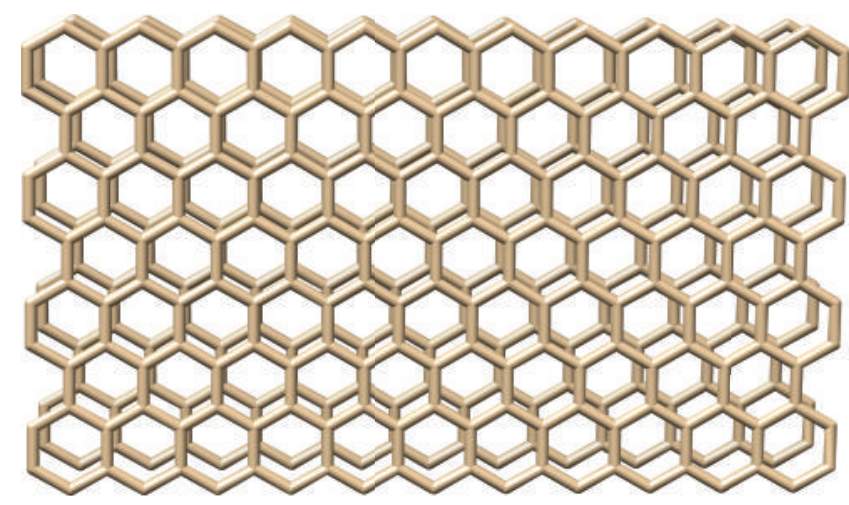

(a)

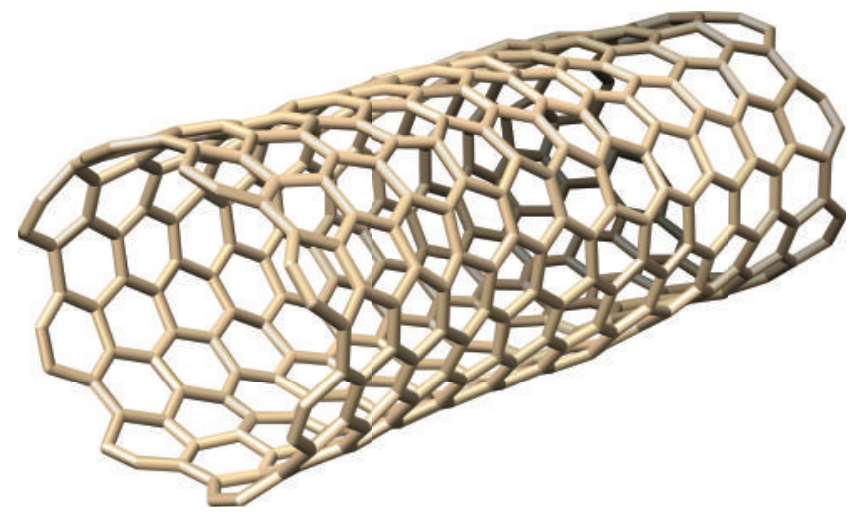

(b)

FIGURE 5: Simulation of armchair nanotubes. The initial (a) and the final (b) structures are shown. The simulation parameters are the following: $1000 \mathrm{~K}$ simulation temperature, $27.05 \AA$ of length, and $15.62 \AA$ of width for the parallel nanoribbons.

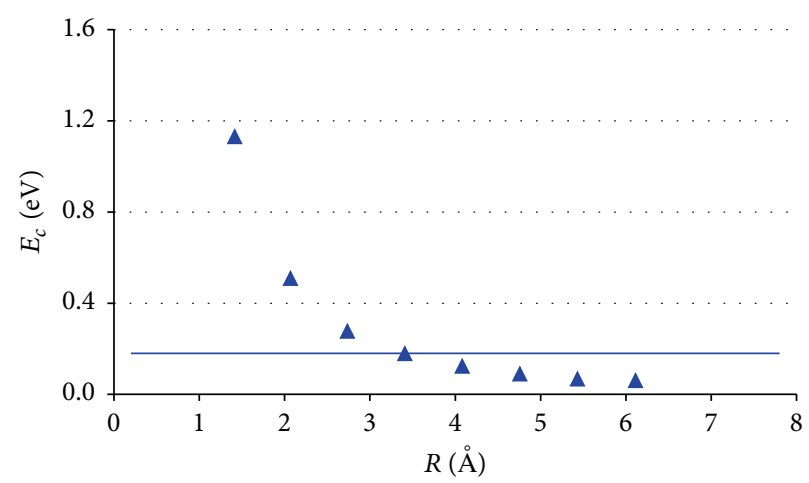

FIGURE 6: The $E_{c}$ curvature energy of armchair nanotubes is shown in the function of the nanotube radius. The horizontal line corresponds to the critical formation energy of $0.18 \mathrm{eV}$.

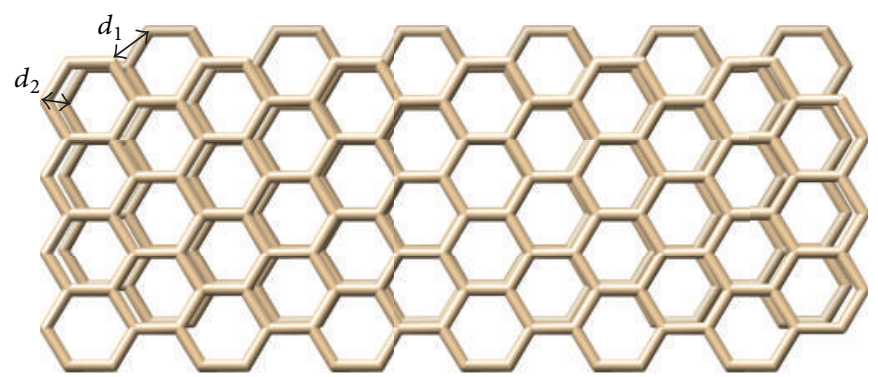

(a)

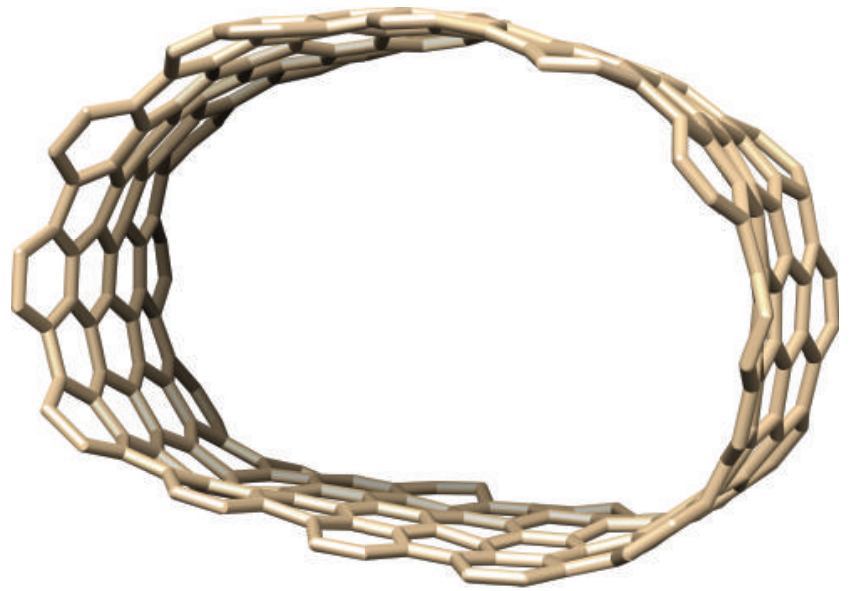

(b)

FIGURE 7: Simulation of zigzag nanotubes. The initial (a) and the final (b) structures are shown. The simulation parameters are the following: $1000 \mathrm{~K}$ simulation temperature, $26.27 \AA$ of length, and $9.84 \AA$ of width for the parallel nanoribbons. The distances between the atoms to be bonded are in order $d_{1}$ and $d_{2}$ at the zigzag and armchair sides of the ribbons. 


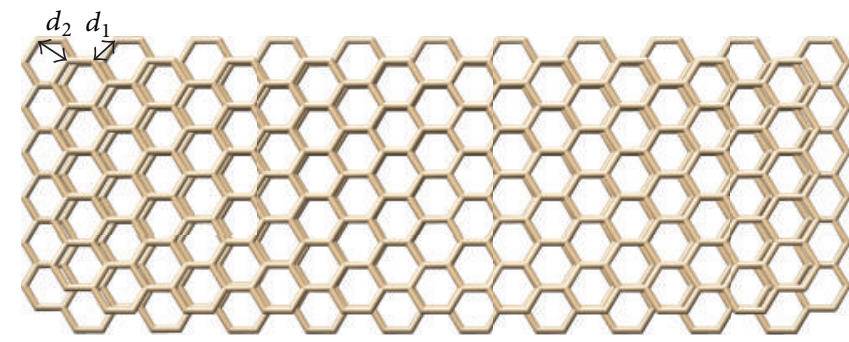

(a)

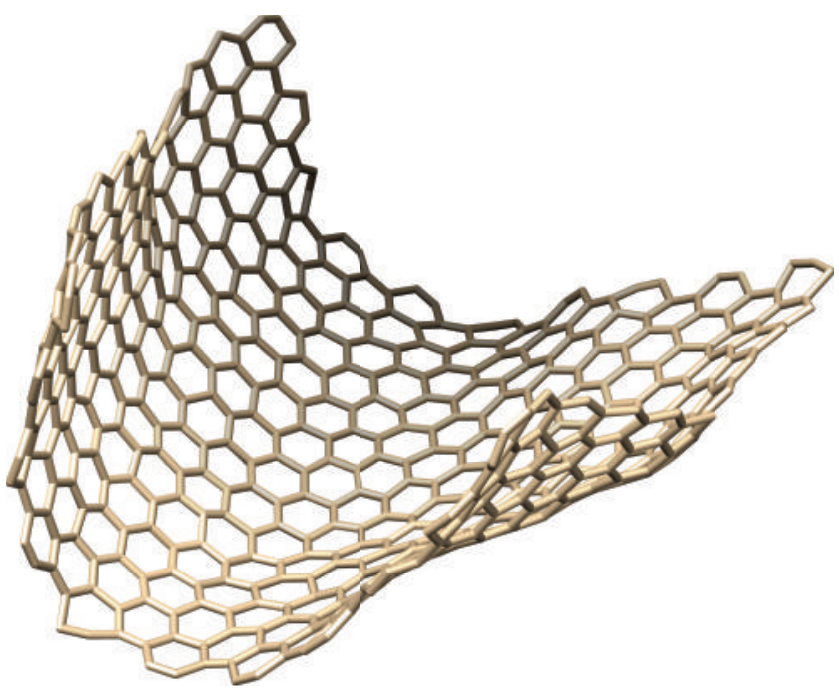

(b)

FIGURE 8: Simulation of zigzag nanotubes. The initial (a) and the final (b) structures are shown. The simulation parameters are the following: $1100 \mathrm{~K}$ simulation temperature, $13.53 \AA$ and $15.99 \AA$ of widths and $41.18 \AA$ and $45.44 \AA$ of lengths for the parallel nanoribbons. The distances between the atoms to be bonded are in order $d_{1}$ and $d_{2}$ at the zigzag and armchair sides of the ribbons.

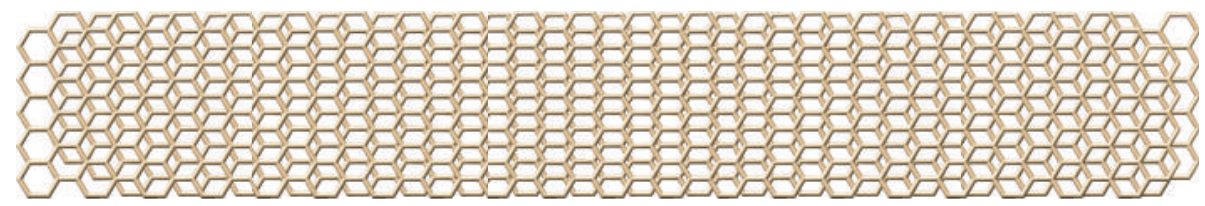

(a)

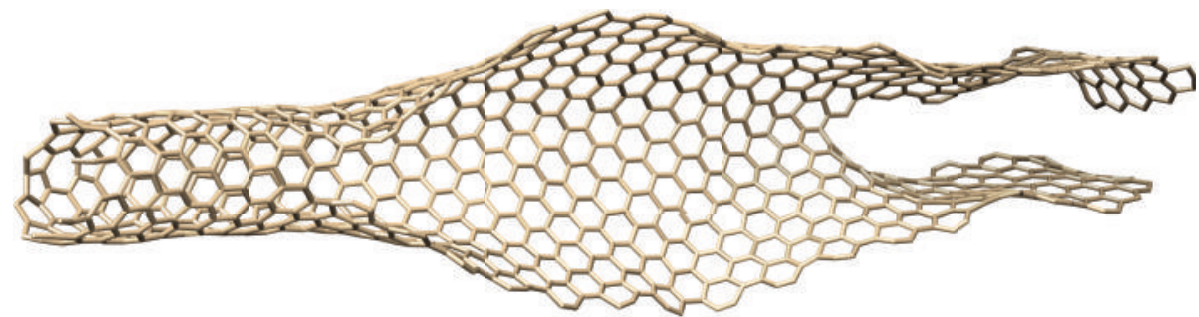

(b)

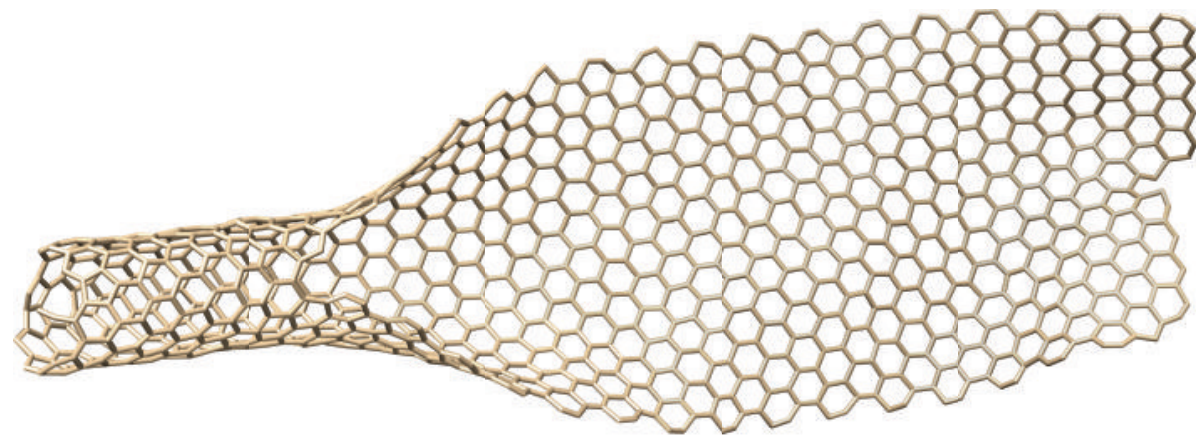

(c)

Figure 9: Simulation of zigzag nanotubes. The initial (a) and the structures after a simulation time of $1.7 \mathrm{ps}$ (b) and $2.8 \mathrm{ps}$ (c) are shown. The simulation parameters are the following: $1000 \mathrm{~K}$ simulation temperature, $85.91 \AA$ of length, and $13.53 \AA$ of width for the parallel nanoribbons. 


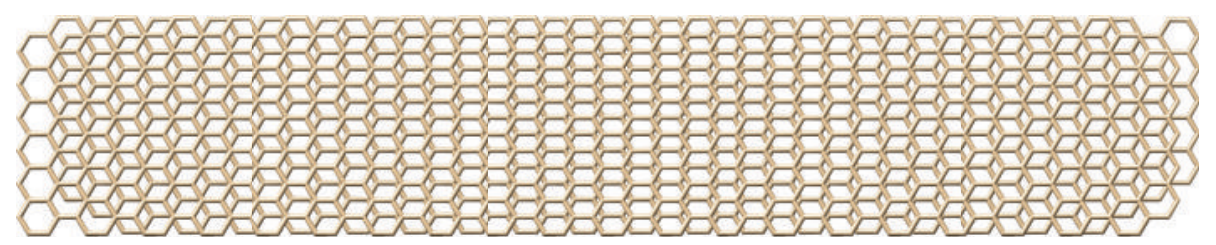

(a)

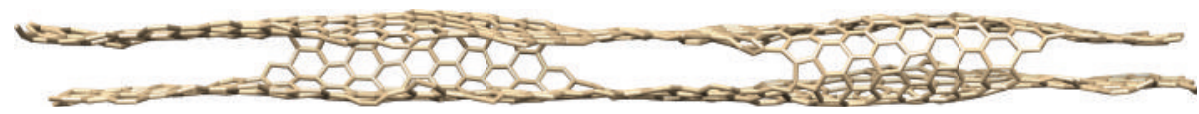

(b)

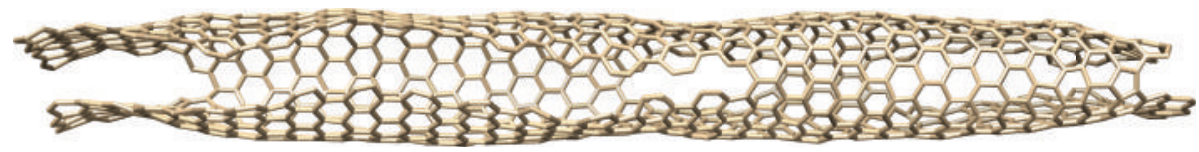

(c)

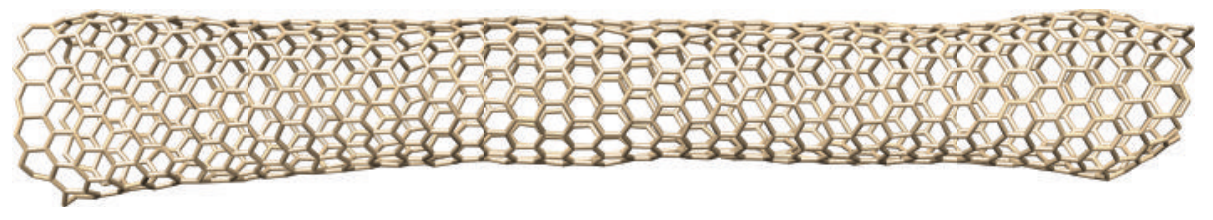

(d)

Figure 10: Simulation of zigzag nanotubes. The initial (a) and the developed structures at $0.45 \mathrm{ps}, 0.77 \mathrm{ps}$, and 2.8 ps are shown. The simulation parameters are the following: $1050 \mathrm{~K}$ simulation temperature, $85.91 \AA$ of length, and $15.99 \AA$ of width for the parallel nanoribbons.

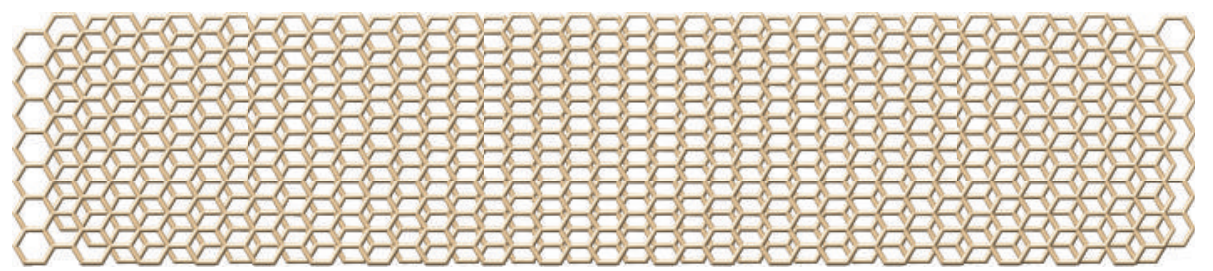

(a)

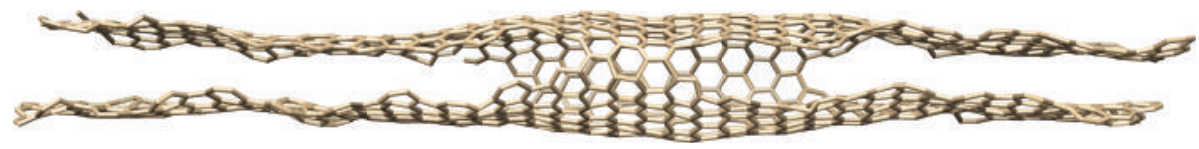

(b)

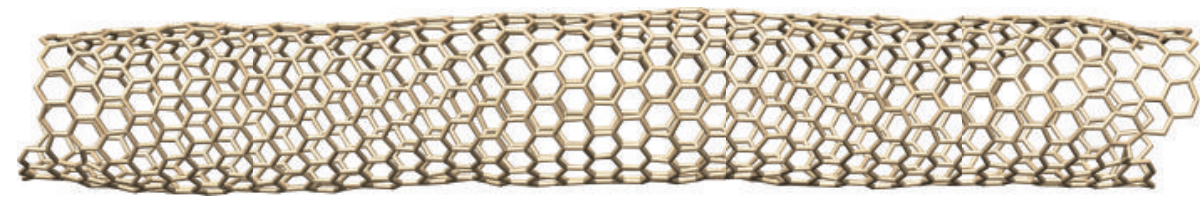

(c)

Figure 11: Simulation of zigzag nanotubes. The initial (a) and the developed structures at 0.8 ps and 2.6 ps are shown. The simulation parameters are the following: $1050 \mathrm{~K}$ simulation temperature, $85.91 \AA$ of length, and $17.92 \AA$ of width for the parallel nanoribbons.

the ribbon are nearer to each other than those at the armchair side. In Figure 7 see the relation $d_{1}>d_{2}$. Here $d_{1}$ and $d_{2}$ are in order of the distances between the atoms to be bonded at the zigzag and at the armchair sides of the ribbons. In order to fulfill the condition of $d_{1}<d_{2}$ at the edges of the ribbons we cut one of them shorter in the following models, as it can be seen in Figure 8.
In the case of zigzag nanotubes the critical ribbon width is greater than the same value at the armchair nanotubes. In the following examples we shall study the problem of the critical width of zigzag nanoribbons. In Figure 8 the widths of the two parallel ribbons are $13.53 \AA$ and $15.99 \AA$. Although the condition of $d_{1}<d_{2}$ is fulfilled, the final structure became a half-sewed configuration. 


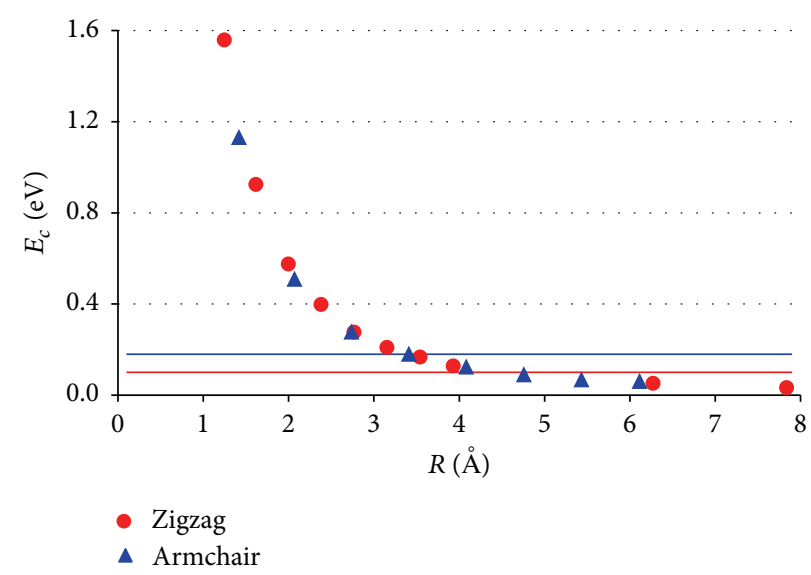

FIGURE 12: The $E_{c}$ curvature energy of armchair and zigzag nanotubes in the function of the nanotube radius is shown. The horizontal line corresponds to the critical formation energy of $0.18 \mathrm{eV}$ for armchair nanotubes and $0.1 \mathrm{eV}$ for zigzag nanotubes.

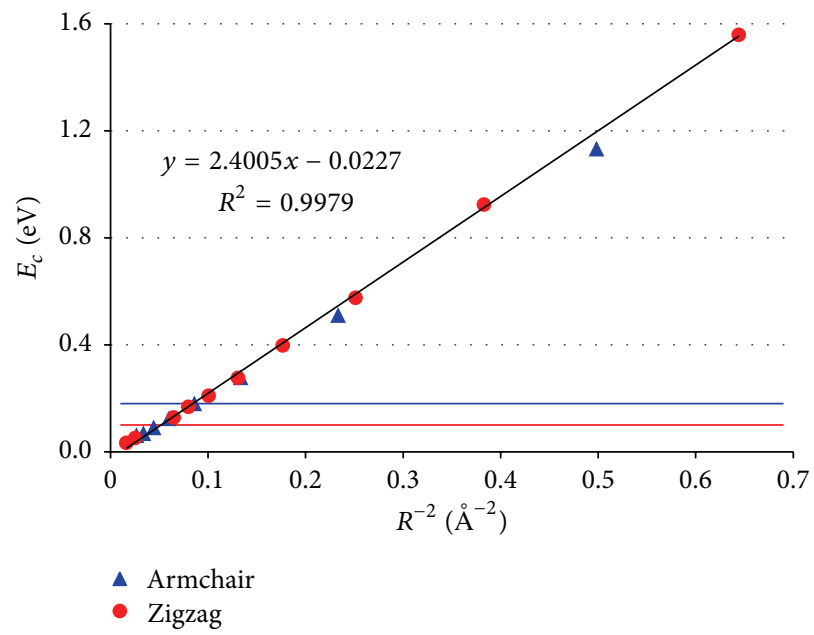

FIGURE 13: The $E_{c}$ curvature energy of armchair and zigzag nanotubes in the function of the square of nanotube curvature $\left(1 / R^{2}\right)$ is shown.

In Figure 9 the two ribbons are shifted in order to obtain the condition of $d_{1}<d_{2}$. We can see the structures after the simulation times of $1.7 \mathrm{ps}$ and $2.8 \mathrm{ps}$. There is still a tendency of constructing a flat structure. In Figure 10 we increased the width to $15.99 \AA$ and the ribbons are still shifted. At $0.4 \mathrm{ps}$ we observed an initial nanotube formation and at $2.8 \mathrm{ps}$ we obtained a perfect zigzag nanotube.

For zigzag nanotubes in the same way as in the case of armchair nanotubes the tube formation process depends strongly on the widths of the ribbons. In Figure 11 we increased the width of the ribbons and the bond formation process started at $0.8 \mathrm{ps}$ reaching the complete tube structure at $2.6 \mathrm{ps}$.

During the formation of zigzag nanotubes we found more difficulties than in the case of armchair nanotubes. We solved the problems by shifting the two ribbons relating one to the other and we obtained larger critical ribbon width than for the armchair nanotubes.

Figure 12 shows the curvature energies of armchair and zigzag nanotubes in the function of the nanotube radius. The two sets of values are practically on the same curve.
The critical ribbon width of zigzag nanotubes is $15.99 \AA$. This corresponds to the nanotube $(14,0)$ and the critical curvature energy of $0.1 \mathrm{eV}$. In Figure 13 the same $E_{c}$ curvature energy of armchair and zigzag nanotubes is presented in the function of the square of nanotube curvature $\left(1 / R^{2}\right)$. We obtained a linear function.

\section{Conclusions}

From our molecular dynamics simulations we obtained the following conditions for nanotube formation from two parallel nanoribbons put one over the other:

(i) For armchair nanotubes the critical ribbon width is $9.23 \AA$ corresponding to the critical curvature energy of $0.18 \mathrm{eV}$.

(ii) For zigzag nanotubes we obtained the critical ribbon width of $15.99 \AA$ and the corresponding critical curvature energy of $0.1 \mathrm{eV}$. 
We obtained linear relationship between the curvature energies and the square of nanotube curvature.

\section{Conflict of Interests}

The authors declare that there is no conflict of interests regarding the publication of this paper.

\section{Acknowledgment}

The support of the grants with identification numbers TÁMOP-4.2.2.A-11/1/KONV-2012-0029 and VKSZ_12-12013-0038 is gratefully acknowledged.

\section{References}

[1] P. Avouris, "Molecular electronics with carbon nanotubes," Accounts of Chemical Research, vol. 35, no. 12, pp. 1026-1034, 2002.

[2] S. J. Tans, A. R. M. Verschueren, and C. Dekker, "Room-temperature transistor based on a single carbon nanotube," Nature, vol. 393, no. 6680, pp. 49-52, 1998.

[3] Y. Zhen, H. W. C. Postma, L. Balents, and C. Dekker, "Carbon nanotube intramolecular junctions," Nature, vol. 402, no. 6759, pp. 273-276, 1999.

[4] K. Keren, R. S. Berman, E. Buchstab, U. Sivan, and E. Braun, "DNA-templated carbon nanotube field-effect transistor," Science, vol. 302, no. 5649, pp. 1380-1382, 2003.

[5] L. Tapasztó, G. Dobrik, P. Lambin, and L. P. Biró, “Tailoring the atomic structure of graphene nanoribbons by scanning tunnelling microscope lithography," Nature Nanotechnology, vol. 3, no. 7, pp. 397-401, 2008.

[6] P. Nemes-Incze, G. Magda, K. Kamarás, and L. P. Biró, "Crystallographically selective nanopatterning of graphene on $\mathrm{SiO}_{2}$," Nano Research, vol. 3, no. 2, pp. 110-116, 2010.

[7] S. S. Datta, D. R. Strachan, S. M. Khamis, and A. T. C. Johnson, "Crystallographic etching of few-layer graphene," Nano Letters, vol. 8, no. 7, pp. 1912-1915, 2008.

[8] Z.-S. Wu, W. C. Ren, L. B. Gao, B. L. Liu, J. P. Zhao, and H. M. Cheng, "Efficient synthesis of graphene nanoribbons sonochemically cut from graphene sheets," Nano Research, vol. 3, no. 1, pp. 16-22, 2010.

[9] L. Jiao, L. Zhang, X. Wang, G. Diankov, and H. Dai, "Narrow graphene nanoribbons from carbon nanotubes," Nature, vol. 458, no. 7240, pp. 877-880, 2009.

[10] Y. Son, M. L. Cohen, and S. G. Louie, "Half-metallic graphene nanoribbons," Nature, vol. 444, no. 7117, pp. 347-349, 2006.

[11] Q. F. Son and X. C. Xie, "Quantum transport through a graphene nanoribbon-superconductor junction," Journal of Physics: Condensed Matter, vol. 21, no. 34, Article ID 344204, 2009.

[12] A. R. Akhmerov, J. H. Bardarson, A. Rycerz, and C. W. J. Beenakker, "Theory of the valley-valve effect in graphene nanoribbons," Physical Review B, vol. 77, no. 20, Article ID 205416, 2008.

[13] M. Y. Han, B. Özyilmaz, Y. Zhang, and P. Kim, "Energy bandgap engineering of graphene nanoribbons," Physical Review Letters, vol. 98, Article ID 206805, 2007.

[14] K. Alam, "Transport and performance of a zero-Schottky barrier and doped contacts graphene nanoribbon transistors,"
Semiconductor Science and Technology, vol. 24, no. 1, Article ID 015007, 2009.

[15] Y. E. Xie, Y. P. Chen, L. Z. Sun, K. W. Zhang, and J. Zhong, "The effect of corner form on electron transport of L-shaped graphene nanoribbons," Physica B: Condensed Matter, vol. 404, no. 12-13, pp. 1771-1775, 2009.

[16] X.-L. Kong and Y.-J. Xiong, "Resonance transport of graphene nanoribbon T-shaped junctions," Chinese Physics Letters, vol. 27, no. 4, Article ID 047202, 2010.

[17] Y.-F. Li, B.-R. Li, and H.-L. Zhang, "The computational design of junctions between carbon nanotubes and graphene nanoribbons," Nanotechnology, vol. 20, no. 22, Article ID 225202, 2009.

[18] Y. P. Chen, Y. E. Xie, L. Z. Sun, and J. X. Zhong, "Asymmetric transport in asymmetric T-shaped graphene nanoribbons," Applied Physics Letters, vol. 93, no. 9, Article ID 092104, 2008.

[19] Z. F. Wang, Q. X. Li, Q. W. Shi et al., "Ballistic rectification in a Zshaped graphene nanoribbon junction," Applied Physics Letters, vol. 92, no. 13, Article ID 133119, 2008.

[20] Y. P. Chen, Y. E. Xie, and J. Zhong, "Resonant transport and quantum bound states in Z-shaped graphene nanoribbons," Physics Letters, Section A: General, Atomic and Solid State Physics, vol. 372, no. 37, pp. 5928-5931, 2008.

[21] D. A. Areshkin and C. T. White, "Building blocks for integrated graphene circuits," Nano Letters, vol. 7, no. 11, pp. 3253-3259, 2007.

[22] D. A. Areshkin and B. K. Nikolić, "I-V curve signatures of nonequilibrium-driven band gap collapse in magnetically ordered zigzag graphene nanoribbon two-terminal devices," Physical Review B, vol. 79, no. 20, Article ID 205430, 2009.

[23] J. Guo, "Modeling of graphene nanoribbon devices," Nanoscale, vol. 4, no. 18, pp. 5538-5548, 2012.

[24] G.-Y. Lee and J. W. Kang, "Controlling resonance frequencies of double-walled carbon-nanotube oscillators with divided outertubes," Journal of Nanoscience and Nanotechnology, vol. 14, no. 8, pp. 6033-6037, 2014.

[25] S. S. Han, K. S. Lee, and H. M. Lee, "Nucleation mechanism of carbon nanotube," Chemical Physics Letters, vol. 383, no. 3-4, pp. 321-325, 2004.

[26] L. He, J.-Q. Lu, and H. Jiang, "Controlled carbon-nanotube junctions self-assembled from graphene nanoribbons," Small, vol. 5, no. 24, pp. 2802-2806, 2009.

[27] I. László and I. Zsoldos, "Graphene-based molecular dynamics nanolithography of fullerenes, nanotubes and other carbon structures," EPL, vol. 99, no. 6, Article ID 63001, 2012.

[28] I. László and I. Zsoldos, "Molecular dynamics simulation of carbon nanostructures: the $\mathrm{C}_{60}$ buckminsterfullerene," Physica Status Solidi B, vol. 249, no. 12, pp. 2616-2619, 2012.

[29] I. László and I. Zsoldos, "Molecular dynamics simulation of carbon nanostructures: the $\mathrm{D}_{5 \mathrm{~h}} \mathrm{C}_{70}$ fullerene," Physica E, vol. 56, pp. 422-426, 2014.

[30] M. P. Allen and D. J. Tildesley, Computer Simulation of Liquids, Clarendon Press, Oxford, UK, 1996.

[31] D. Frenkel and B. Smit, Understanding Molecular Simulation, Academic Press, San Diego, Calif, USA, 1996.

[32] D. Porezag, T. Frauenheim, T. Köhler, G. Seifert, and R. Kaschner, "Construction of tight-binding-like potentials on the basis of density-functional theory: application to carbon," Physical Review B, vol. 51, no. 19, pp. 12947-12957, 1995.

[33] L. Verlet, "Computer "experiments" on classical fluids. I. Thermodynamical properties of Lennard-Jones molecules," Physical Review, vol. 159, no. 1, pp. 98-103, 1967. 
[34] S. Nosé, "A molecular dynamics method for simulations in the canonical ensemble," Molecular Physics, vol. 52, no. 2, pp. 255268, 1984.

[35] W. G. Hoover, "Canonical dynamics: equilibrium phase-space distributions," Physical Review A, vol. 31, no. 3, pp. 1695-1697, 1985. 

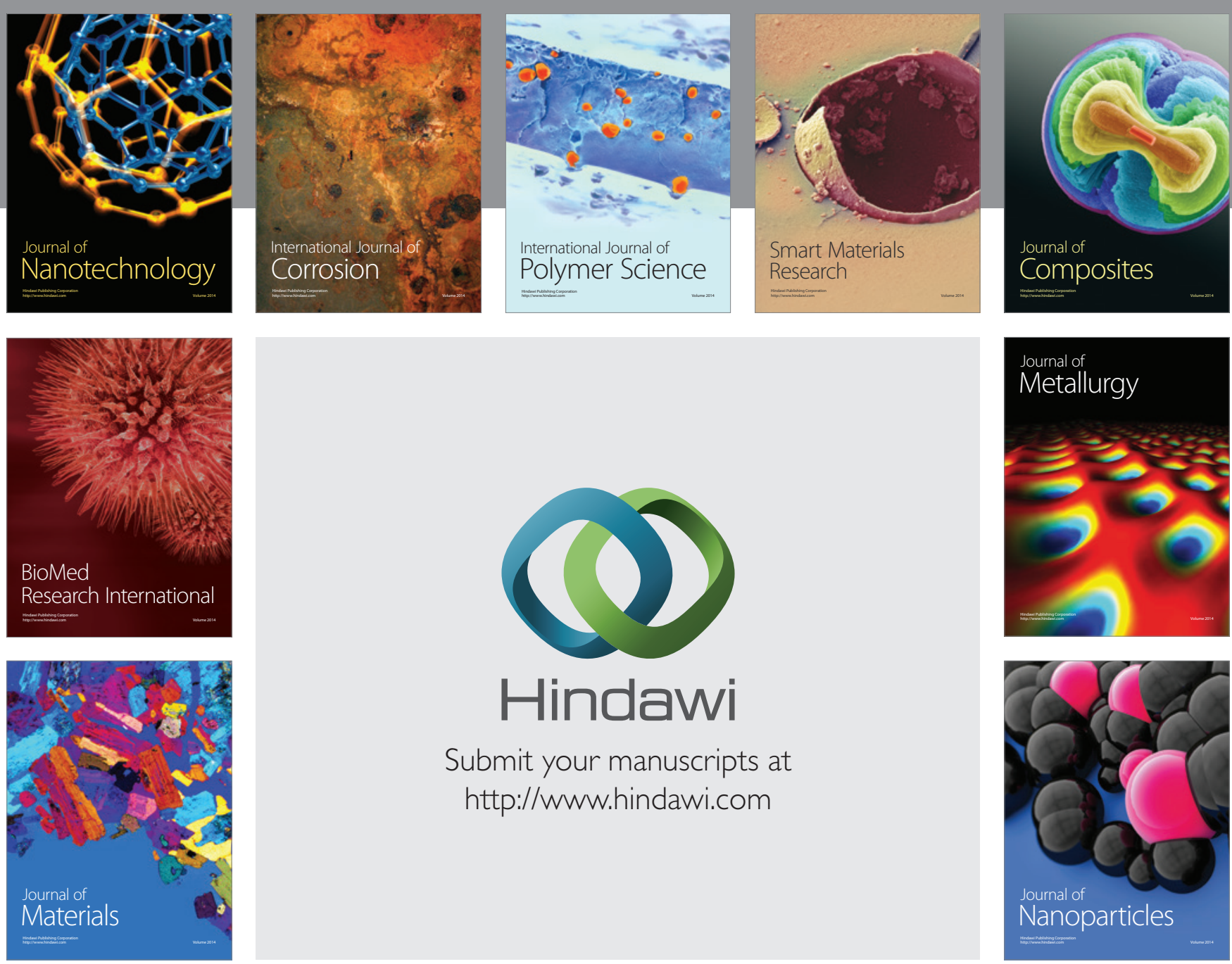

Submit your manuscripts at http://www.hindawi.com
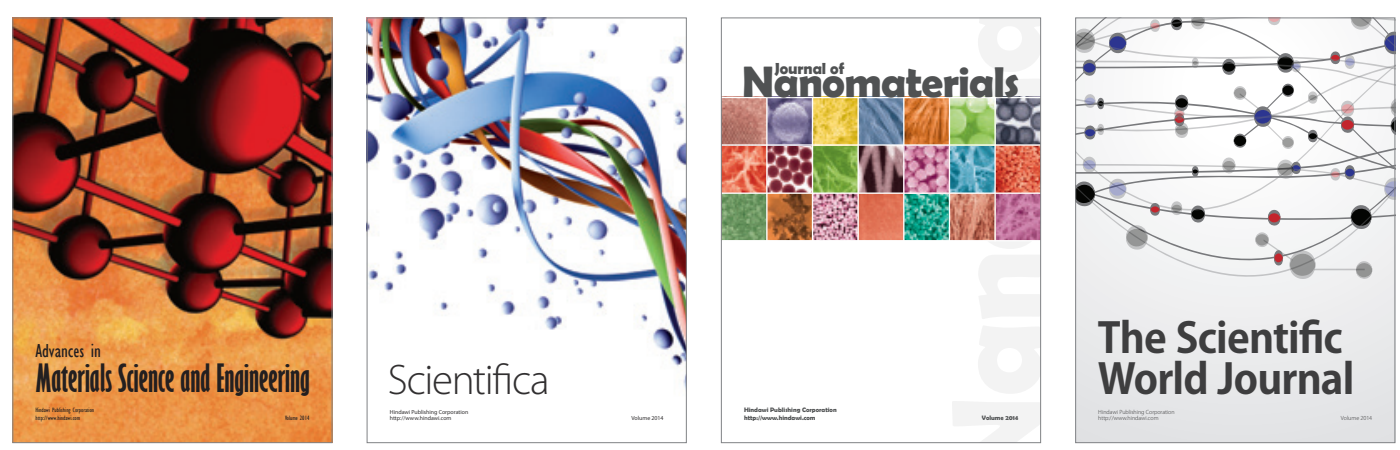

\section{The Scientific World Journal}
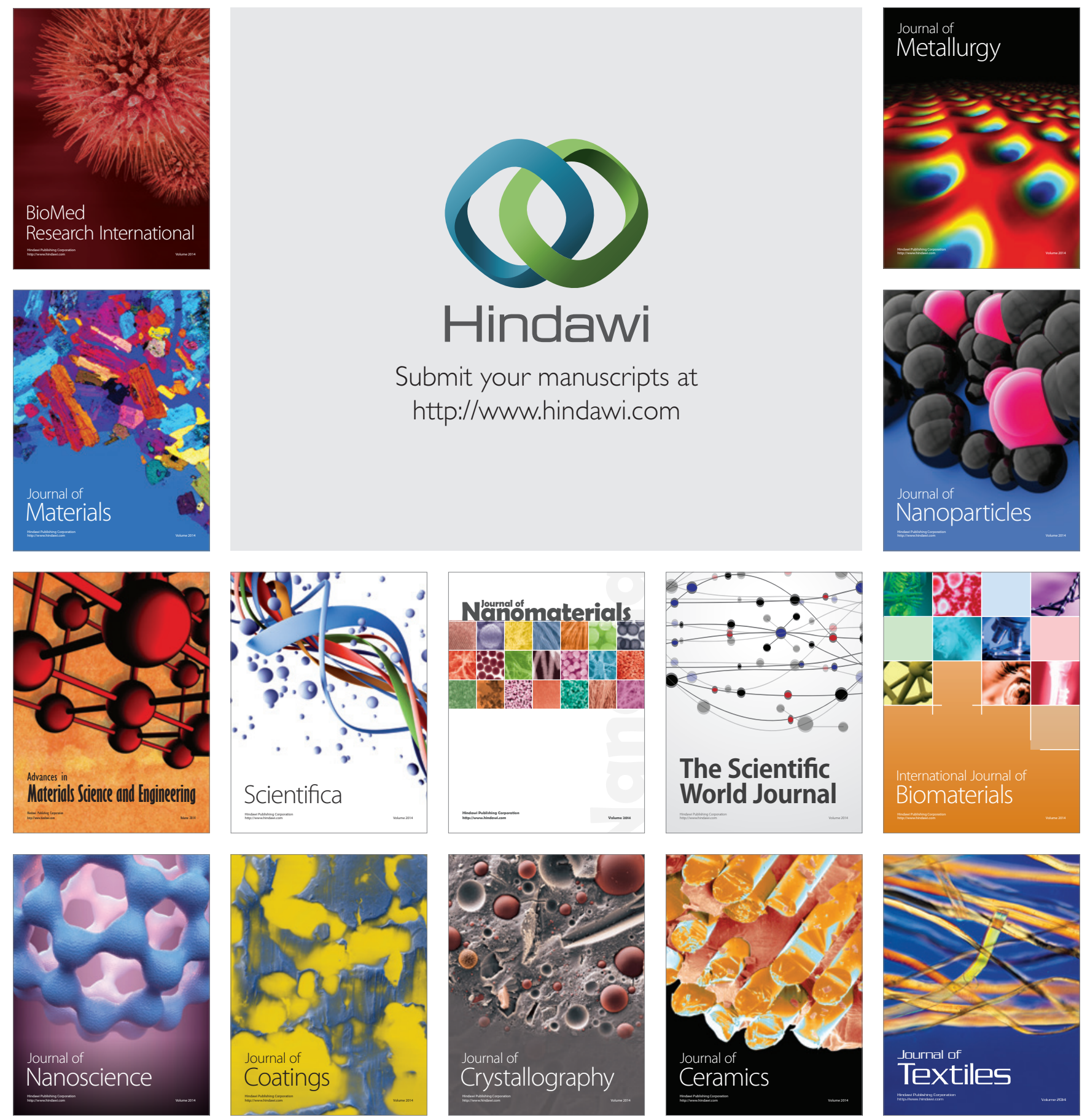